\title{
Incompetencia cervical: diagnóstico y tratamiento
}

\author{
Alvaro Monterrosa Castro*
}

RESUMEN: La incompetencia cervical es una causa relativamente poco frecuente de pérdida gestacional, que se considera se ha sobrediagnosticado, más sinembargo es una importante razón para aborto repetido en el segundo trimestre de la gestación. El diagnóstico debe sustentarse en los antecedentes obstétricos y tratar de evidenciar su existencia de forma preconcepcional para simultáneamente descartar otras causas de pérdidas repetidas. Alcanzada la gestación y de forma temprana debe realizarse cerclaje cervical, utilizando técnica de Shirodkar o McDonald, que presentan iguales ratas de supervivencia fetal e iguales ratas de complicaciones. En nuestra experiencia hemos tenido buenos resultados con técnica de McDonald y seda en forma de trenza, como material de sutura, pasando la rata de supervivencia fetal del $10 \%$ pre-cerclaje al $88 \%$ post-cerclaje. En aquellas pacientes en que no se puede realizar cerclaje cervical, se recomienda cerclaje cérvico-istmico vía abdominal.

PALABRAS CLA VES: Cerclaje cervical, incompetencia cervical, técnica de McDonald, técnica de Shirodkar, cerclaje cérvico istmico abdominal, aborto espontáneo repetido, aborto habitual, parto pretérmino.

SUMMARY: Cervical is a relatively litle common cause of gestatorial loss, which is considered to have been diagnosticated, nevertheless, it is an important reason for repeted abortion in the second trimester of gestation. Diagnostic should be based on proceding diagnostic and try to evidence its existence in a preconceptional way to simultaneously discord other causes of repeted losses. As gestation takes place and in an early stage cervical cerclaje should be practiced using either Shirodkar or McDonald technique which show equal rates of complication. In our practice we have had good results withMcdonald Tecnique and thread in braid way as suture material increasing the rate of fetua survival from $10 \%$ re cerclaje to $88 \%$ post-cerclaje. On those patients who can not take cervical cerclaje, abdominal cervical isthmical cerclaje is recommended.

KEY WORDS: Cervical cerclaje, cervical incompetence, McDonald technique, Shirodkar technique, abdominal cervical isthmic cerclaje, repeted spontaneus abortion, habitual abortion, preterm birth.

Riverius, Culpepern y Cole 1658 relacionaron pérdida fetal habitual con defectos cervicales, y Gream en 1865 utilizó el término incompetencia cervical (1).

\section{Definición}

Incompetencia cervical es la incapacidad del cérvix uterino para mantener el embarazo in-útero hasta el término, debido a defectos anatómicos o funcionales (23 ), siendo frecuente la pérdida de la gestación en el segundo trimestre (4). La incompetencia cervical es factor de riesgo a parto prematuro, y éste frecuenta causa de morbi-mortalidad perinatal (5). Cuando el embarazo alcanza un peso crítico, se vence la resistencia del orificio cervical, se protruyen las membranas amnióticas y posterior a un corto e indoloro trabajo de parto se produce expulsión fetal (6).

\section{Incidencia}

Existe gran controversia en la frecuencia de la incompetencia cervical con respecto al número de partos normales (7-8). Weissman et al (9) informan que la

* Instructor. Departamento de Ginecología y Obstetricia. Facultad de Medicina. Universidad de Cartagena, Colombia. incompetencia cervical complica el $0.05 \%$ al $1.8 \%$ de todos los embarazos, es causa del 16 al $20 \%$ de todas las pérdidas gestacionales del segundo trimestre $(2,9-10)$. Ocurre en el $0.2 \%$ de todos los abortos (2) y es responsable del 25\% de los partos pretérminos (11).

\section{Etiología}

No se conoce con certeza la importancia relativa de cada uno de los factores etiológicos, los cuales pueden ser:

(A) Adquiridos, lesión anatómica producida por traumatismo obstétrico: dilatación y legrado o parto instrumentado $(2,7,10)$, o por cirugía ginecológica, generalmente conización del cérvix (12). Las lesiones adquiridas se consideraban la mayor causa de debilidad en el esfínterinterno cérvico-uterino (6). (B) Congénitos, defectos histológicos en cuello macroscópicamente intacto (9-10). Palmer y Lacomme en 1948 (7) sospechan causa congénita en mujeres nulíparas, por la existencia de debilidad en la musculatura del extremo superior del cuello y del orificio cervical interno (6).

(C) Fisiológicos, cuando la dilatación es secundaria a irritabilidad uterina (contracciones) anormalmente persistente (3). (D) Anatómicos, por distorsión morfológica uterina, sea por malformación uterina congénita o por miomas (13). 


\section{Diagnóstico}

Por no disponerse de un método fiable y objetivo, no existen criterios bien definidos para el diagnóstico de incompetencia cervical (13). La historia de los embarazos previos es muy importante y decisiva para sospechar la entidad. Las pacientes suelen ser multigestantes con uno o más abortos en el segundo trimestre (14). Se debe interrogar sobre antecedente de partos precipitados, partos instrumentados, dilatación y legrado (7). Es importante el antecedente de cauterización o conización cervical (2, 12). Artículos de Shirodkar (15) y McDonald (16) señalan la historia típica de incompetencia cervical: pérdida del embarazo en el segundo trimestre, ruptura prematura de membranas sin actividad uterina dolorosa, trabajo de parto breve y expulsión de feto usualmente vivo. En una revisión nuestra (17), 100\% de las pacientes eran multigestantes, $100 \%$ tenían abortos en el segundo trimestre y el $82.3 \%$ habían sido previamente dilatadas para legrado, datos similares a los de McDonald: $95.5 \%, 93.3 \%$, $68.8 \%$ respectivamente (6). No existe un método exacto que confirme el cuello incompetente (13-14, 18), basándose el diagnóstico en los antecedentes obstétricos (6-7). Nada sustituye a la historia obstétrica en el diagnóstico de la incompetencia cervical $(1,19)$. Debe realizarse de forma preconcepcional para adelantar manejo preventivo. Cuando el diagnóstico no se ha realizado, y antes de la ruptura de membranas, debido a la dilatación cervical la paciente suele experimentar peso en hemiabdomen inferior y la sensación de plenitud vaginal. A la especuloscopia se observa el cuello parcialmente dilatado o la presencia de membranas que salen por el orificio cervical.

\section{Estudios auxiliares de diagnóstico}

Ningún estudio, realizado antes o durante la gestación es preciso para confirmar el diagnóstico.

El Test de Hegar se realiza estando la paciente no grávida, se evalúa el canal cervical, utilizando dilatadores de Hegar, siendo la prueba positiva el paso fácil del dilatador No. 8 (2-3,20). La prueba de tracción del catéter, o de la sonda de Foley, consiste en colocar y llenar con 1 cc el balón de la sonda de Foley y luego traccionar para evidenciar la laxitud y relajación del orificio cervical interno $(2,20)$.

Histerosalpingografía (14), para medir el orificio cervical interno, se ha utilizado y cifras superiores a seis $\mathrm{mm}$ son sugestivas de incompetencia cervical (3), aunque el método puede tener numerosos falsos positivos y falsos negativos (2). Recientemente Hricak et al (21) destacan el valor que puede tener la resonancia nuclear magnética.

En la paciente embarazada, la ultrasonografía se ha incluido como método auxiliar de diagnóstico $(11,14,22-$ 23). Nzeh y Adetoro (14) encontraron mediciones del diámetro del orificio cervical interno en pacientes con incompetencia cervical de $16 \mathrm{~mm}$ a $22.5 \mathrm{~mm}$ desde la 10 a 27 semanas de gestación, mientras que en población control de mujeres sanas tenían diámetros entre 7.7 a 14 $\mathrm{mm}$ en igual intervalo gestacional. Existe evidencia ultrasonográfica de estar incrementando el diámetro del orificio cervical interno en pacientes con incompetencia cervical $(11,14)$. Los mismos autores (14) citan a Brook et al, quienes aseveran que el diámetro del orificio cervical interno mayor de $19 \mathrm{~mm}$, puede ser indicativo de incompetencia cervical. Otros (24) consideran importante la medición seriada ultrasonográfica de la longitud del cérvix en el embarazo, para fijar el riesgo de parto pretérmino. Consideran valor normal de $52 \pm 12 \mathrm{~mm}$ hasta las 34 semanas de gestación. La ultrasonografía vaginal es valiosa en la valoración de estas pacientes (29). Zlatnik y Burmeinstaer (20) estudiaron con histerosalpingografía, test de Hegar y prueba de tracción del catéter en pacientes no embarazadas con antecedentes de pérdidas en el segundo trimestre de la gestación y establecen que la elasticidad cervical puede tener valor predictivo en el éxito de un embarazo posterior.

\section{Tratamiento}

Los tratamientos para esta condición han sido quirúrgicos (7), colocando una sutura alrededor del cuello incompetente. En 1902 Herman (26) realiza con éxito traquelorrafía en mujeres con aborto habitual. En 1922, Child (27) reporta el valor de la sutura para preservar el orificio cervical interno en las mujeres con abortos repetidos. Palmer y Lacomme (28) en 1948 informan un caso exitoso, retirando una cuña en la pared anterior del istmo, cerca alorificio interno y la sutura de los bordes con catgut. Lash y Lash (29) realizan resección romboidal a nivel cérvico-vaginal, seguido de reparación del defecto, reportan casos en 1950 (29) y otros en 1957 (30), consiguiendo embarazos en el $60 \%$ y productos viables en el $50 \%$ de ellas. Sin embargo una cuarta parte de las pacientes no pudieron tener nueva gestación (10). En 1955, Shirodkar (15) describe su técnica en la paciente embarazada, vía vaginal, levantando la mucosa del cérvix y suturando con una tira de fascia lata para cerrar el cérvix dilatado, reportó éxitos en el $79 \%$ de sus casos. La técnica continúa siendo de gran valor $(2,7,18)$. En 1957 McDonald (16) describe una técnica usando hilo de mersilene y puntos en "bolsa de tabaco" circundando el cuello, por vía vaginal y con la paciente gestante. Técnica sencilla de amplio uso en la actualidad (2, 9-10-, 16, 18, 31). Barker tuvo buenos resultados con la técnica de McDonald con tira de fascia lata y posteriormente con el uso de cinta de mersilene, Page manejó cinta de Catgut y tira de gasa de Oxycel (10). En 1965 Benson y Durfee (32) propusieron el cerclaje transabdominal cérvico-itsmico, colocando cinta de Mersilene al nivel de los útero-sacros y cardinales, teniendo la paciente de 10-14 semanas de gestación, con tasas de supervivencia fetal superior al $90 \%$ (32-36), pero más riesgos reales y potenciales que las técnicas vaginales, sobre todo de hemorragia, mayor estancia hospitalaria y la obligatoriedad de cesárea $(2,32-37)$. Hoy día el cerclaje cérvico-itsmico transabdominal se relega para casos con cerclajes cervicales previos fallidos o para cérvix con severo desgarro (2-3, 32-37).

El cerclaje cervical es el método quirúrgico más usado para prevenir la pérdida habitual en el segundo trimestre debido a incompetencia cervical, debe ser electivo, aplicado antes que se presenten cambios en el cuello (17), siendo de elección la técnica de McDonald o Shirodkar $(7,15-16)$, procedimientos que tienen iguales 
índices de supervivencia fetal $(2-3,4,6,19,38)$. Diversos materiales de sutura han sido utilizados (6). Está definido que el material debe ser no absorbible (3, 4142).: Mersilene o hiladillo. Seda, trenzando varias hebras y usando técnica de McDonald, la proponemos (17) como un buen material de sutura, con iguales altas tasas de supervivencia perinatal e iguales bajas tasas de complicaciones que las encontradas con otros materiales $(2,18$, 38). El cerclaje se puede realizar exitosamente como cirugía ambulatoria (17), lo mismo aseveran Wetchler et al (43) y Trehan et al (44). El momento oportuno para la cirugía son las 14 semanas (17), lo cual permite el diagnóstico previo de anormalidades del huevo o errores de implantación (45). Ayhan et al (18) encontraronsupervivencia fetal del $74 \%$ si se interviene antes de las 12 semanas, $84 \%$ entre 12 y 18 semanas y $68 \%$ después de las 18 semanas. Entre más avanzada sea la edad gestacional, se puede encontrar mayor grado de borramiento cervical y dilatación, con menores posibilidades de éxito.

El cerclaje de urgencia, se realiza cuando hay modificaciones cervicales y/o protrusión de membranas, pueden no tener los mismos niveles de supervivencia fetal $(2,10$, 38-39), o como sugieren Novy et al (40): requerir úteroinhibición, posición de Trenbdelemburg, antibióticos parenterales, aminocentesis y reducción de las membranas protruidas. A esta condición no se debe permitir que lleguen las pacientes con antecedentes de abortos repetidos en el segundo trimestre.

Deben proscribirse por completo hoy en día las técnicas de cerclaje preconcepcional $(7,17)$.

\section{Complicaciones del manejo quirúrgico}

Con la técnica de McDonald puede formarse un anillo fibrótico en el cérvix a nivel del cerclaje, pero suele ser tan delgado que rara vez constituye obstáculo para la dilatación plena del cuello $(6,9)$. La incidencia de cesárea suele ser igual a otras condiciones médicas u obstétricas (10, 14). La Distocia cervical es común complicación de falla en la progresión del trabajo de parto y aumenta las tasas de cesárea (11).

La complicación más grave puede ser el fracaso de la cirugía, por inicio de contractilidad uterina en el postoperatorio inmediato o ruptura accidental de las membranas durante el procedimiento (18).

La infección en la línea de sutura, absceso, corioamnionitis, sepsis, pudieran presentarse $(19,46)$. Aunque el cérvix puede ser reservorio de agentes potencialmente patógenos, existen pocos reportes de infecciones graves como complicaciones del cerclaje cervical (8). No hay diferencia en la incidencia de sepsis entre las técnicas de Shirodkar y de McDonald (8). Charles y Edwards (8) en su revisión informan mayor riesgo de corioamnionitis como complicación infecciosa del cerclaje, cuando éste es aplicado después de 19 semanas de gestación, lo cual puede ser debido a la presencia de infección focal en las membranas por mayor área de exposición a la microflora cérvico-vaginal. La flora vaginal y su reacción inflamatoria liberan proteasas, colagenasas, prostaglandinas y linfoquinas que favorecen la actividad uterina, rompen las fibras de colágena e inducen maduración cervical (40). La manipulación quirúrgica puede ser un factor facilitador de migración bacteriana. Pacientes con dilatación cervical en elsegundo trimestre tiene una mayor frecuencia de invasión microbiana a la cavidad amniótica. Romero et al (46) encontraron una prevalencia del $51 \%$, siendo los gérmenes más frecuentes: el Ureaplasma Urealítucum, la Gardnerella Vaginalis, la Candida Albicans y la Fusobacterias sp. La tasa de infección es mayor en cerclajes de urgencias, que en electivos $(10,38)$. Nuestra experiencia con 17 pacientes intervenidos de forma electiva, no tuvimos ningún caso de infección severa (17). Si la paciente presenta actividad uterina, fiebre y taquicardia posterior al cerclaje, sobre todo si fue después de las 19 semanas de gestación, debe realizarse amniocentesis y cultivo para descartar corioamninitis (8). Si el cultivo es negativo, tiene menor pronóstico que si es positivo (46). La incidencia de corioamnionitis posterior a cerclaje es del 1 a $5 \%$ (3).

Uso de antibióticos profilácticos y hormonas para minimizar el riesgo infeccioso y la irritabilidad uterina, son medidas sugeridas por algunos autores (17) y cuestionadas por otros $(2,7,44)$. Harger (1) dice que no hay pruebas de que estas medidas perioperatorias tengan algún beneficio. Se prohíben los coitos y se limita la actividad física durante el resto de la gestación $(17,19)$.

La más frecuente complicación tardía es la actividad uterina de pretérmino y la ruptura prematura de membranas (18). Es frecuente la actividad uterina prematura (3, 18). Estudios tocográficos suelen señalar contracciones esporádicas o aumento transitorio del tono basal (7). Son de valor el uso de agentes tocolíticos, para prevenir la instalación del trabajo de parto pretérmino $(3,5,47)$.

Laceraciones cervicales y ruptura uterina han sido descritas en la literatura (7). Entre nuestros 17 casos no tuvimos estas complicaciones graves (17). Raras complicaciones como fístula útero-vaginal y vesico vaginal han sido reportadas (2).

\section{Contraindicaciones para cerclaje cervical}

El cerclaje cervical está contraindicado en: ruptura prematura de membranas, sangrado uterino, dilatación mayor de cuatro centímetros, borramiento total cervical, gran desgarro cervical, infección cérvico-vaginal severa, anomalías fetales conocidas (3).

\section{Eficacia del cerclaje cervical}

Con el cerclaje cervical profiláctico, técnica de Shirodkar o McDonald, cabe esperar una frecuencia de productos vivos entre 80 y $90 \%(8,17-19,45)$. Si el cerclaje se realiza de forma tardía, cuando ya hay dilatación marcada del cérvix o con protrusión de las membranas, cerclaje de urgencia, las tasas de éxito son menores al $50 \%(6,18-19,38-39)$. Más sin embargo, Novy et al (40) tienen experiencias con técnica de Shirodkar, en pacientes con estados avanzados de dilatación cervical y protrusión de membranas, reportando tasas de éxito superiores al $80 \%$.

La eficacia del procedimiento ha sido evaluado por la rata de supervivencia fetal post-cerclaje, con la rata 
de supervivencia fetal de los embarazos previos al cerclaje, en las mismas pacientes (18). En nuestra serie (17) la rata de supervivencia post-tratamiento fue del $88.2 \%$, comparada con el $10.3 \%$ pre-tratamiento. Datos similares son expuestos por muchos autores $(2-3,8,18$ -
19, 38). Esta metodología evaluativa es cuestionada. No existen trabajos prospectivos, controlados que sustenten la validez del cerclaje, pero las observaciones indirectas enunciadas sugieren que pueden ser soluciones adecuadas (2).

\section{BIBLIOGRAFIA}

1. Harger HJ. Cervical cerclaje: patient, selection, morbidity and success rates. Clin. Perinatol. 1983; 10: 321-330.

2. Golan A., Barnan R., Wexler S. Incompetence of the uterine cervix Obst. Gynecol. Survey 1989; 44(2): 96-107.

3. Shortle B., Jewelewicz R. Cervical incompetence. Fertil Steril 1989 52(2): 181-188.

4. MRC/RGOC. Final Report of the medical research council. Royal collegue of obstetricians and gynecologists multi center randomised trial of cervical cerclaje. British J Obstrical Gynaecol. 1993; 100: 516-523.

5. Monterrosa A. Incidencia de parto pretérmino y factores de riesgo. Rev. Col. Obstet. Ginecol. 1991; 42(3): 199-207.

6. McDonald IA. Cerclaje cervical. Ginecología y Obstetricia. Temas actuales. 1980; 3: 461-478

7. Fuste P, Deulofen P, Martínez JM et al. Cerclaje cervical con técnica deShirodkar: indicaciones, eficacia y repercusión sobre la gestación. Estudio de 56 casos. Clin. Invest. Gin. Obst. 1993; 20(10): 437-442.

8. Charles D., Edwards WR. Infection complications of cervical cerclaje. Am. J. Obstet. Gynecol. 1981; 141: 1065-1070.

9. Weissman A., Jakobi P., Zahi S. The effect of cervical cerclage on the course of labor. Obstet. Gynecol. 1990; 76(2): 168-171.

10. Austin KL. Cerclaje cervical. Rev. Med. Panamá. 1993; 1892: 79-87.

11. Michaels WH., Montgomery C., Kard J et al. Ultrasound differentiation of the competente from the incompetent cervix: pregnation of preterm delivery. Am. J. Obstet. Gynecol. 1986; 154: 537-541.

12. Larsson G., Grendesell, Gullberg B et al. Outcome of pregnancy after conization. Act. Obst. Gynecol. Scand. 1982; 61: 461-464.

13. Jewelewicz R. Incompetent cervix. Pathogenesis, diagnosis and treatment. Semin. Perinatol. 1991; 15(2): 156-161.

14. Nzeh DA., Adetoro OO. Sonographic assessment of the competent cervix in pregnancy. Int. J. Gynecol. Obstet. 1992; 37: 179-184.

15. Shirodkar UN. A new method of operative treatment for habitual abortion in the second trimestre of pregnancy. Antiseptic 1955; 52: 229-235.

16. McDonald IA. Suture of the cervix for inevitable miscarriage. J. Obstet. Gynaecol Br Empire 1957; 63(3): 346.

17. Monterrosa A. Cerclaje Cervical con trenza de seda y técnica de McDonald. Unimetro. In Prensa.

18. Ayhan A., Mercan R., Tuncer ZC et al. Post concepcional cervical cerclaje. Int. J. Gynecol. Obstet. 1993; 42: 243-246, 352.

19. Branch W. Intervenciones quirúrgicas para la insuficiencia cervical. Clínicas Obstétricas y Ginecológicas 1986; 2: 305-324

20. Zlatnik FJ, Burmeinster LF. Interval evolution of the cervix for predicting pregnancy outcome and diagnosing cervical incompetence. J. Reprod. Med. 1993; 38(5): 365-369.

21. Hricak H., Chang YC., Cann CE et al. Cervical incompetence Preliminary evaluation with MR imaging. Radiology 1990; 174: 821826.

22. Michaels WH., Schereiber FR., Podgett RJ et al. Ultrasound surveillance of the cervix in term gestations: management of cervical incompetency. Obst. Gynecol. 1991; 78(5): 739-744.

23. O'Lary JH., Ferrel RE. Comparison of ultrasonographic and digital cervical evaluation. Obstet. Gynecol. 1986; 68: 718-724.

24. Ayers JWT., DeGrood RM., Compton Ea et al. Sonographic evaluation of cervical length in pregnancy of preterm cervical effacement in patients atriesk for premature delivery. Obstet. Gynecol. 1988; 71: 939-944.
25. Chung TK., Hoines CJ., Kon D et al. Transvaginal sonography in the diagnosis and management of cervical incompetence. Gynecol. Obst. Invest 1993; 36(1): 59-61.

26. Herman GE. Notes on emmets operation as a prevention of abortion. J. Obstet. Gynaecol. Br. Commounw 1902; 2: 256.

27. Chil GG. Sterility and concepcion appleton century crofts New York, 1922; 174-187.

28. Palmer R., Lacomme M. Ladeance de l'orifice interne cause d'avortemenst a repetition une observation de dechirire cervico isthnique reporce chirirgicalement avec cesation a terme consecutive. Gynecol. Obstet. 1948; 47: 905.

29. Lash AF., Lash A. Habitual abortion. The incompetent internal os of the cervix. Am. J. Obstet. Ginecol. 1950; 59(1): 68-72.

30. Lash Af., Lash A. Incompetent internal os of the cervix. Diagnosis and treatment. Am. J. Obstet. Gynecol. 1957; 79: 346-350.

31. McCulloch B., Bergen S., Pielet B et al. McDonald cerclaje under pudendal nerve block. Am. J. Obstet. Gynecol. 1993; 168(2): 499502.

32. Benson RC., Durfee RB. Transabdominal cervico-uterine cerclaje during pregnancy for the treatment of cervical incompetency Obstet. Gynecol. 1965; 25: 145-149.

33. Mahrah $\mathbf{M}$. Transabdominal cervical cerclaje during pregnancy. A modified technique. Obstet. Gynecol. 1987; 502: 502.

34. Watkin RA. Transabdominal cervico uterine suture. Aust Nz J Obstet. Gynaecol. 1972; 12: 62.

35. Novy JM. Transabdominal cervico istmic cerclage for the management of repetitive abortion and premature delivery. Am. J. Obstet. Gynecol. 1982; 143: 44.

36. Van-Donget PW., Nijhuis JG. Transabdominal cerclege. Eur J. Obstet. Gynecol. Reprod. Biol. 1991; 41(2): 97-104.

37. Novy JM. Transabdominal cervico-istmic cerclage: a reapproisal 25 year after ist introduction. Am. J. Obstet. Gynecol. 1991; 164(6): $1635-1642$.

38. Treadwel MC., Bonsteen RA., Bottoms SF. Prognostic factors and complication rates for cervical cerclage: a review of 482 cases. Am. J. Obstet. Gynecol. 1991; 165(3): 555-558.

39. McDougall J., Siddle N. Emergency cervical cerclaje. Obst. Gynecol. Survey 1992; 46(6): 379-380.

40. Novy MJ., Haymond J., Nicholson M. Schirodkar cerclaje in amultifactorial approach to the patient with advanced cervical changes. Am. J. Obstet. Gynecol. 1990; 162(6): 1412-1420.

41. Golomb J., Ben-Chaim JS., Goldwasser B et al. Conservative treatment of a vesicocervical fistula resulting from Shirodkar cervical cerclage. J. Urol. 1993; 149(4): 833-834.

42. Ansan Ah., Reynolds RA. Cervical incompetence. A reviews. J. reprod. Med. 1987; 32: 161-170.

43. Wetchler BV., Brick J. Safety of outpatient cerclaje. J. Reprod. Med. 1990; 35: 243-246.

44. Trehan Ak., Kenney A., Fergusson IL. Outpatient cervical cerclaje. The Lancet 1992; 339(13): 1482

45. Angel JL., Pietrantoni M., O'Brien WF et al. Cervical cerclaje in early pregnancy. J. perinatol. 1991; 11(3): 258-261.

46. Romero R., González R., Sepúlveda W. Infection and labor. VIII. Microbial invasion of cervical incompetence: prevalence and clinical significance. Am. J. Obstet. Gynecol. 1992; 167 (4, pt 1): 1086-1091.

47. Cromblehome WR., Monkoff HL., Delke I et al. Cervical cerclaje: an aggresive approach to threatened or recurrent pregnancy wastage. Am. J. Obstet. Gynecol. 1983; 146: 168-172. 